\title{
Interview
}

\section{Schleswig-Holstein: Countdown zur Pflegekammer}

\author{
Auch die Pflegekammer Schleswig-Holstein nimmt Gestalt an. Über die \\ Vorbereitungen zur Wahl sprachen wir mit Patricia Drube, Vorsitzende \\ des Errichtungsausschusses der Pflegekammer.
}

? Frau Drube, der Countdown zur Wahl der 1. Kammerversammlung hat begonnen. Was ist die Kammerversammlung und wie können sich Pflegende beteiligen?

Drube: Die Kammerversammlung ist das Entscheidungsorgan der Kammer. Sie besteht aus 40 gewählten Vertreterinnen und Vertretern unserer Berufsgruppe und bestimmt, wie die Kammer ihre gesetzlichen Aufgaben ausführt. Sie entscheidet, was die Berufsordnung regelt, über den Haushalt und über die Höhe der Kammerbeiträge. Sie bestimmt auch, welche Fortbildungsverpflichtungen es geben wird und was die Kammer unternimmt, um die Arbeitsbedingungen zu verbessern. Die Mitglieder der Kammerversammlung sind Pflegefachpersonen, die hauptberuflich ihren Pflegeberuf ausüben und ehrenamtlich das Mandat in der Kammerversammlung wahrnehmen. Die operative Arbeit, z.B. die Mitgliederverwaltung und die Organisation von Sitzungen, übernehmen die hauptamtlichen Mitarbeiter der Geschäftsstelle der Pflegeberufekammer.

\section{? Welche Fristen gilt es einzuhalten?} Drube: Die Kammer besteht aus den drei Berufsgruppen Altenpflege, Gesundheitsund Krankenpflege und Gesundheitsund Kinderkrankenpflege. Die Angehörigen dieser drei Gruppen wählen jeweils Vertreterinnen und Vertreter aus ihrer eigenen Berufsgruppe in die Kammerversammlung. Kandidatenvorschläge können nur in Form von Listen gemacht werden: Dafür müssen sich Personen aus der gleichen Berufsgruppe zusammentun. Weil die Wahl zwischen dem 1. März und dem 3. April 2018 statt findet, müssen Wahlvorschläge spätestens am 1. Februar 2018 in der Geschäftsstelle des
Errichtungsausschusses vorliegen. Alle Wahlberechtigten bekommen per Post die Wahlvorschläge(listen) ihrer Berufsgruppe und ihres Wahlkreises zugesandt. Am 4. und 5. April findet die Stimmauszählung statt. Am 6. April muss das Ergebnis feststehen, damit die konstituierende Sitzung am 21. April in Neumünster stattfinden kann.

? Was bedeutet es für Pflegende, sich in der Kammer zu engagieren?

Drube: Ich treffe viele Kollegen, die sehr konkret sagen können, was aus ihrer Sicht wie verändert werden müsste, damit Pflegende bessere Arbeitsbedingungen und Pflegebedürftige eine bessere Versorgung hätten. Durch ein Engagement in der Pflegeberufekammer haben sie die Möglichkeit, ihre Ideen und Forderungen dort auszusprechen und weiterzugeben, wo sie gesellschaftliche und politische Entscheidungen beeinflussen können. Die ehrenamtliche Kammerarbeit bedeutet, von unserem Mitbestimmungsrecht Gebrauch zu machen, dafür zu sorgen, dass wir die Art unserer Berufsausübung wieder selbstbestimmt gestalten. Dass es sich um eine ehrenamtliche Tätigkeit handelt, schreckt einige ab. Fahrtkosten werden jedoch selbstverständlich erstattet, für die Teilnahme an Sitzungen eine Aufwandsentschädigung gezahlt. Wir gehen davon aus, dass die Kammerversammlung 2018 ca. fünf Sitzungen haben wird. Wer mehr tun will, kann Ausschüsse unterstützen, z.B. zum Thema Berufsordnung, Haushalt, Beitragsordnung, Fortbildung oder Öffentlichkeitsarbeit.

? Was ist Ihnen besonders wichtig bei der Einrichtung der Pflegekammer?

Drube: In der Vergangenheit haben wir immer wieder die Erfahrung gemacht

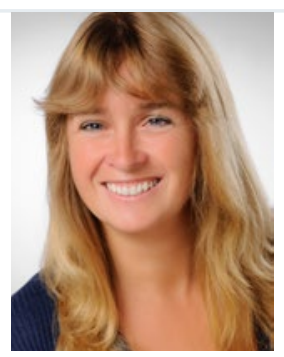

Patricia Drube

Vorsitzende des Errichtungsausschusses der Pflegekammer

fremdbestimmt zu werden. Unsere berufliche Handlungskompetenz wurde zunehmend eingeschränkt. Deshalb fällt es vielen schwer zu glauben, dass dies bei der Pflegeberufekammer anders ist - zumal es sich um eine Pflichtmitgliedschaft handelt. In diesem Fall ist die Pflichtmitgliedschaft gerade deshalb erforderlich, um den Prozess demokratisch zu gestalten. Nur wenn alle Kollegen Mitglied sind, können sie sich an der Wahl beteiligen und mitbestimmen. Aus meiner Sicht bedeutet berufliche Professionalität auch, Verantwortung für die Gestaltung unserer Berufsausübung zu übernehmen und dort mitzureden, wo unsere Kompetenz im Hinblick auf eine Verbesserung unserer eigenen beruflichen Situation und der Versorgungssituation gefragt ist. Die Kammer gibt uns dazu eine neue Möglichkeit.

? Wie viele Pflegende wird die Pflegekammer vertreten?

Drube: Wir haben derzeit ca. 21.000 Pflegefachpersonen registriert und gehen davon aus, dass es bis zu 30.000 werden könnten.

? Was sind die nächsten Schritte? Drube: Am 21. April wird sich die Kammerversammlung konstituieren und dann unverzüglich ihre Arbeit aufnehmen. Am 8. Mai wird der Landespflegekongress in Kiel stattfinden. Dort wird sich die Pflegeberufekammer präsentieren und vielleicht schon etwas dazu sagen können, welche Themen sie als erstes angehen wird.

Das Interview führte Sabine Kempa. Das vollständige Interview lesen Sie online auf www.springerpflege.de. 